\title{
Total Synthesis of the Cyclic Dodecapeptides Wewakazole and Wewakazole B
}

\author{
Martyn Inman, Hannah L. Dexter and Christopher J. Moody* \\ School of Chemistry, University of Nottingham, University Park, Nottingham NG2 7RD, U.K. \\ Supporting Information Placeholder
}

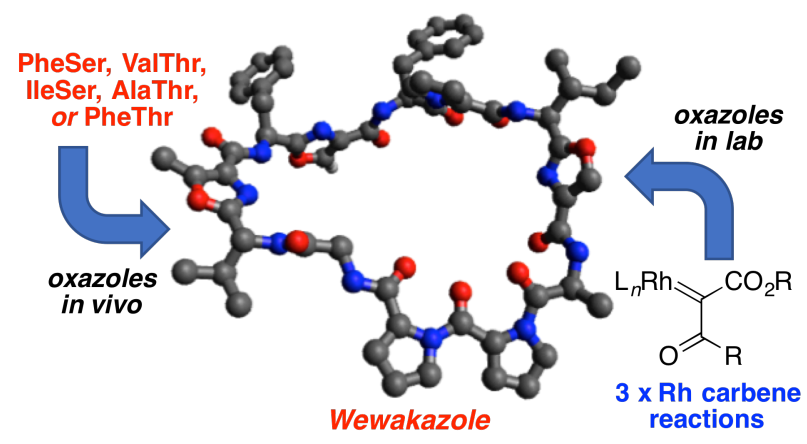

\begin{abstract}
The cyclic dodecapeptides wewakazole and wewakazole B have been synthesized by a divergent strategy via a common tris-proline containing oxazole octapeptide and two separate bisoxazole containing tetrapeptide units, followed by peptide coupling and macrocyclization. The three oxazole amino acid fragments are readily accessible by rhodium(II)-catalysed amide $\mathrm{N}-\mathrm{H}$ insertion of diazocarbonyl compounds, or by the cycloaddition of rhodium carbenoids with nitriles.
\end{abstract}

Wewakazole 1 was isolated from the cyanobacterium Lyngbya majuscula off the coast of Papua New Guinea in 2003, and assigned a modified cyclododecapeptide structure incorporating three prolines, and in which one threonine and two serine residues had been cyclized to form oxazoles. ${ }^{1}$ Although no biological activity was reported, subsequent studies showed that it was active against non-small cell lung cancer H-460 cells. ${ }^{2}$ Thirteen years later, the structurally related wewakazole B 2 was reported from a different genus of cyanobacteria, Moorea producens, in the Red Sea. ${ }^{3}$ Wewakazole B was of particular interest, given its reported anticancer activity $\left(\mathrm{IC}_{50} 0.58 \mu \mathrm{M}\right.$ against $\mathrm{MCF} 7$ breast cancer cells), ${ }^{3}$ and the scarcity of material for further biological evaluation. Although two total syntheses of wewakazole B have recently been reported, ${ }^{4,5}$ its mode of action remains unknown. Given our previous work in the synthesis of post-translationally modified peptides, and particularly those containing azoles, ${ }^{6-10}$ these two structures caught our attention as attractive targets for total synthesis.

The two wewakazole structures differ only by the presence or absence of a methyl group on one oxazole and the identity of two of the peptide residues: the 5-unsubstituted oxazole, phenylalanine and valine present in wewakazole are replaced with a 5-methyloxazole, alanine and phenylalanine in wewakazole B. As all these variations are found within one portion of the structures, a divergent strategy for the synthesis of the two compounds presented itself, whereby the common octapeptide $\mathbf{3}$ could be synthesized and coupled to the two bis-oxazole containing tetrapeptide units $\mathbf{4}$ and 5. An additional advantage of this approach is that macrocyclization can be carried out by amide coupling of the $N$-terminal to a $C$ terminal glycine, reducing the steric bulk around the reactive center and minimizing the risk of epimerization in what will inevitably be a highly dilute, and hence slow, macrocyclization step. We have previously reported the use of rhodium(II)-catalyzed amide $\mathrm{N}-\mathrm{H}$ insertion of diazocarbonyl compounds for 
the synthesis of 5-substituted oxazoles, ${ }^{11,12}$ as well as the cycloaddition of metallocarbenoids with nitriles for the synthesis of 5-unsubstituted oxazoles. ${ }^{13,14}$ Our retrosynthetic plan employs both these reactions, allowing the wewakazoles to be retrosynthesized to their component $L$-amino acids, and two diazocarbonyl compounds 6 and 7 (Scheme 1). A protecting group strategy using methyl and ethyl $C$ terminal esters and $N$-terminal Boc groups was chosen, as the required intermediates ought to be otherwise inert to the deprotection conditions for these groups.

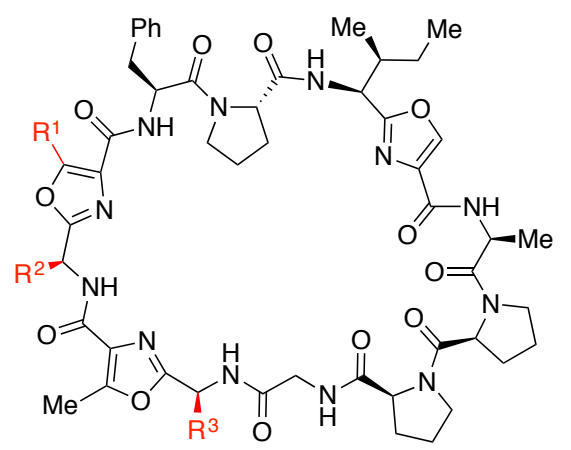

1 wewakazole; $\mathrm{R}^{1}=\mathrm{H}, \mathrm{R}^{2}=\mathrm{CH}_{2} \mathrm{Ph}, \mathrm{R}^{3}=\mathrm{CHMe}_{2}$

2 wewakazole $\mathrm{B} ; \mathrm{R}^{1}=\mathrm{R}^{2}=\mathrm{Me}, \mathrm{R}^{3}=\mathrm{CH}_{2} \mathrm{Ph}$

Figure 1. Structures of the cyclododecapeptides wewakazole and wewakazole B highlighting structural differences.

Scheme 1. Retrosynthesis of the wewakazoles.
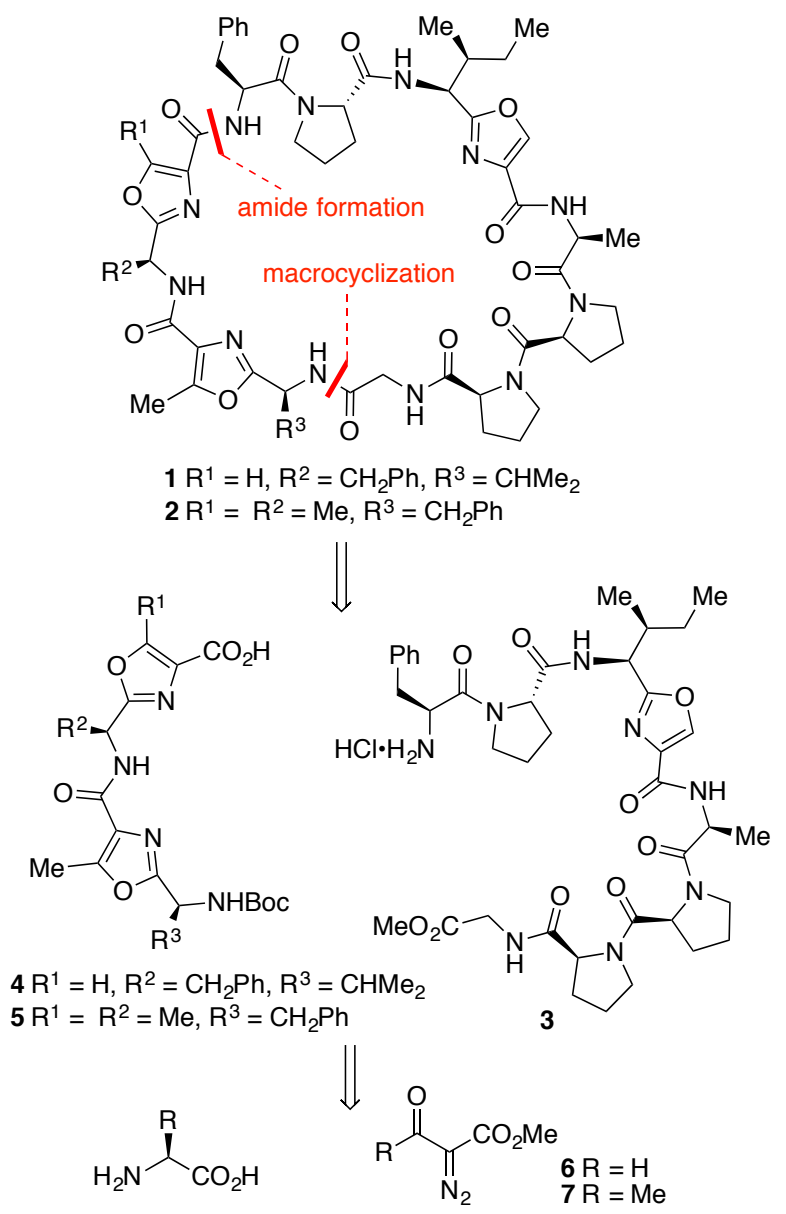
The synthesis began with the construction of the common octapeptide fragment 3 . Coupling of Bocalanine to proline methyl ester, and of Boc-proline to glycine methyl ester, both proceeded without difficulty using $N, N, N^{\prime}, N^{\prime}$-tetramethyl-O-(1H-benzotriazol-1-yl)uronium hexafluorophosphate (HBTU) and $N, N$-diisopropylethylamine (DIPEA) to give dipeptides 8 and 10. Upon removal of both proline protecting groups, the coupling of these acid $\mathbf{9}$ and amine $\mathbf{1 1}$ fragments proved more difficult; HBTU gave very poor results, but the use of $N$-(3-dimethylaminopropyl)- $N$-ethylcarbodiimide hydrochloride (EDCI) and 1-hydroxybenzotriazole (HOBt) gave the desired tetrapeptide 12 in 80\% yield (Scheme 2). Deprotection of the Boc-group in tetrapeptide 12 was only carried out immediately prior to its further reaction, as the hydrochloride salt $\mathbf{1 3}$ was hygroscopic. The presence of a Pro-Pro linkage generally caused rotameric behavior to be observed in the NMR spectra of these intermediates, but this could be minimized by the use of deuterated DMSO as the solvent. The use of variable temperature NMR further reduced the occurrence of rotamers, although this was not performed routinely on all the rotameric intermediates.

Scheme 2. Synthesis of first tetrapeptide fragment.

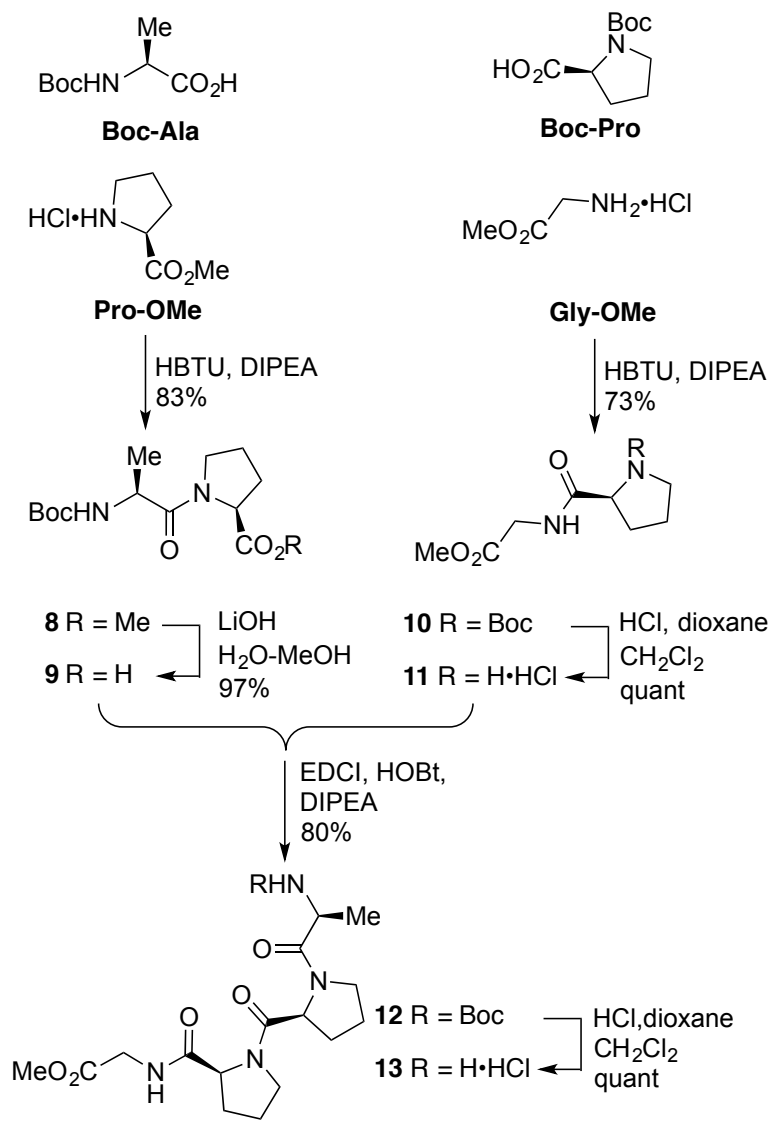

Concurrently, Boc-isoleucinamide was dehydrated with diethyl chlorophosphate to give the nitrile 14. Slow addition of diazo compound 6 to a solution of the nitrile and $\mathrm{Rh}_{2}(\mathrm{pfm})_{4}$ in chloroform at reflux gave only a $30 \%$ yield of the oxazole, but with recovery of $65 \%$ of the nitrile giving an $86 \%$ yield based on recovered starting material. Attempts to increase the conversion beyond this led to much lower recovery of material. The oxazole $\mathbf{1 5}$ was treated with $\mathrm{HCl}$ in dioxane to give the hydrochloride salt 16. Finally, Boc-phenylalanine was coupled to proline methyl ester with HBTU to give dipeptide 17, and the ester was hydrolyzed to give the carboxylic acid 18. Coupling of amine $\mathbf{1 6}$ and acid 18 gave the tetrapeptide 19, hydrolysis of which gave the carboxylic acid 20 (Scheme 3).

The coupling of the two tetrapeptide units 13 and $\mathbf{2 0}$ proved challenging; EDCI gave only 30\% yield, while reagents such as 1-[bis(dimethylamino)methylene]-1H-1,2,3-triazolo[4,5-b] pyridinium 3-oxide 
hexafluorophosphate (HATU), HBTU, benzotriazol-1-yl-oxytripyrrolidinophosphonium hexafluorophosphate (PyBOP) and 2,4,6-tripropyl-1,3,5,2,4,6-trioxatriphosphorinane-2,4,6-trioxide (T3P) offered little improvement. Eventually, it was found that the use of 4-(4,6-dimethoxy-1,3,5-triazin-2-yl)-4methylmorpholinium chloride (DMTMM) ${ }^{15}$ in THF gave a satisfactory $77 \%$ yield, despite the poor solubility of the coupling agent in that solvent. This reaction could be carried out on gram scale in $67 \%$ yield, permitting access to large quantities of the common octapeptide fragment 3 .

Attention then turned to the synthesis of the two bis-oxazole fragments 4 and $\mathbf{5}$. The wewakazole A fragment 4 was synthe-sized via the phenylalanine-derived nitrile 22, which underwent cycloaddition with diazo compound $\mathbf{6}$ to give oxazole $\mathbf{2 3}$ in 38\% yield, again with significant recovery of the nitrile (43\%), followed by Boc deprotection. A solution of Boc-valinamide and $\mathrm{Rh}_{2}(\mathrm{OAc})_{4}$ in refluxing chloroform was subjected to dropwise addition of diazo compound 7, giving the intermediate $\mathrm{N}-\mathrm{H}$ insertion product in moderate yield. ${ }^{6}$ Cyclodehydration under Wipf's conditions ${ }^{16}$ using triphenylphosphine, iodine and triethylamine gave the oxazole 25 in good yield, and treatment with lithium hydroxide gave the carboxylic acid 26. Coupling amine $\mathbf{2 4}$ with acid $\mathbf{2 6}$ using HATU gave the desired bis-oxazole $\mathbf{2 7}$ in excellent yield, followed by hydrolysis to yield the wewakazole A fragment 4.

Scheme 3. Synthesis of second tetrapeptide fragment.

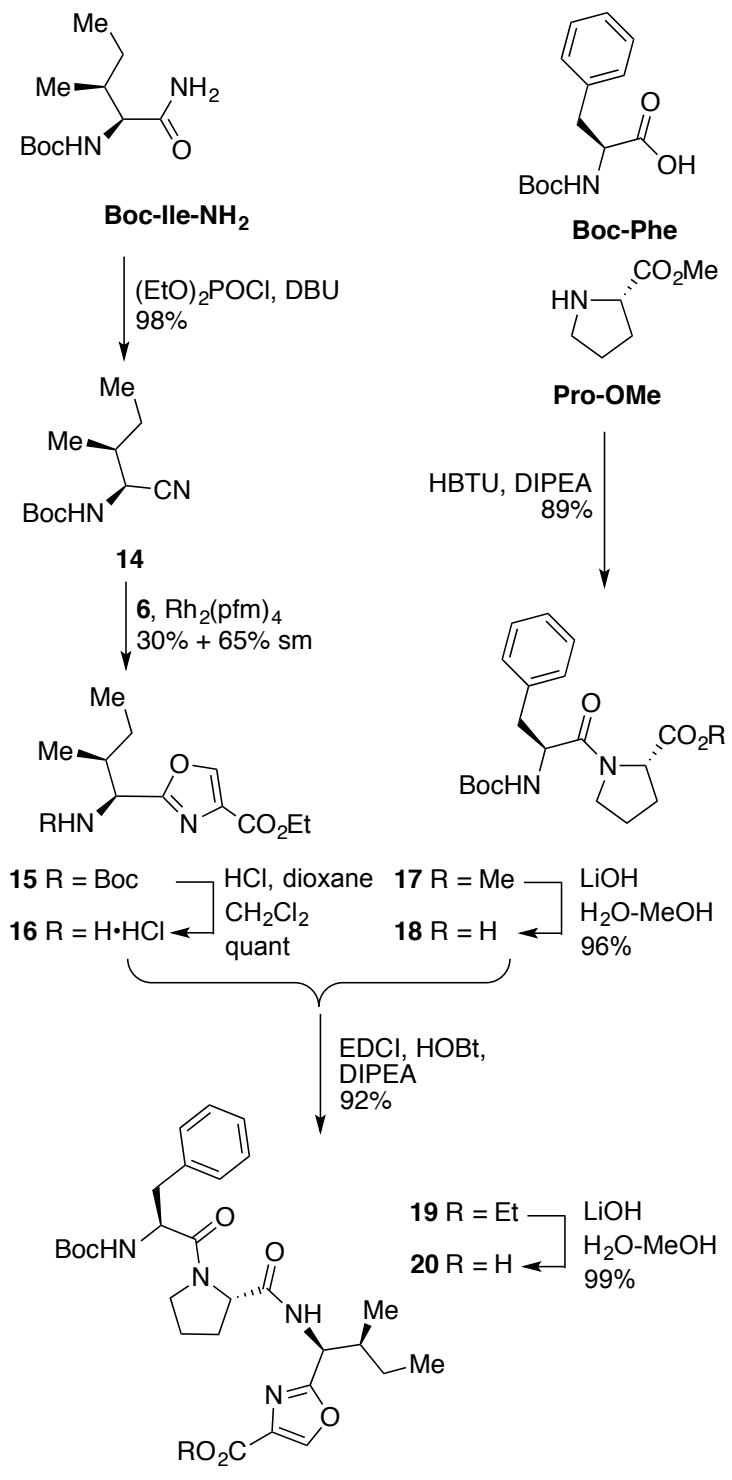


Scheme 4. Synthesis of the common octapeptide 3.

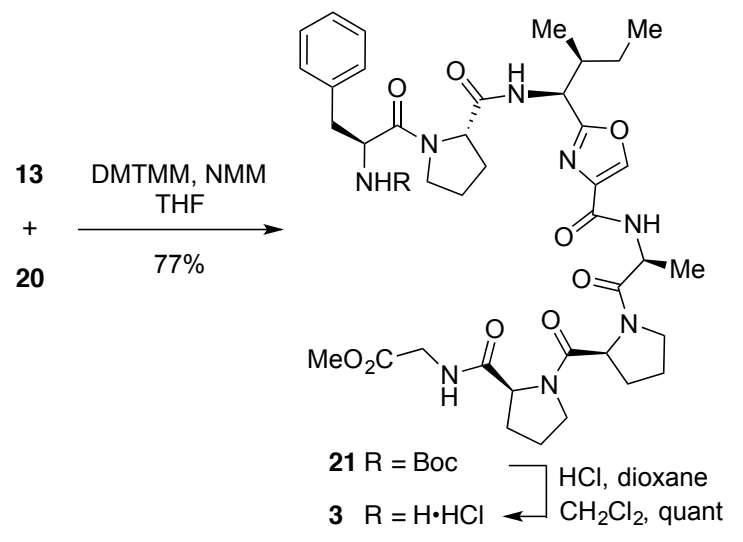

Scheme 5. Synthesis of bis-oxazole fragment of wewakazole.

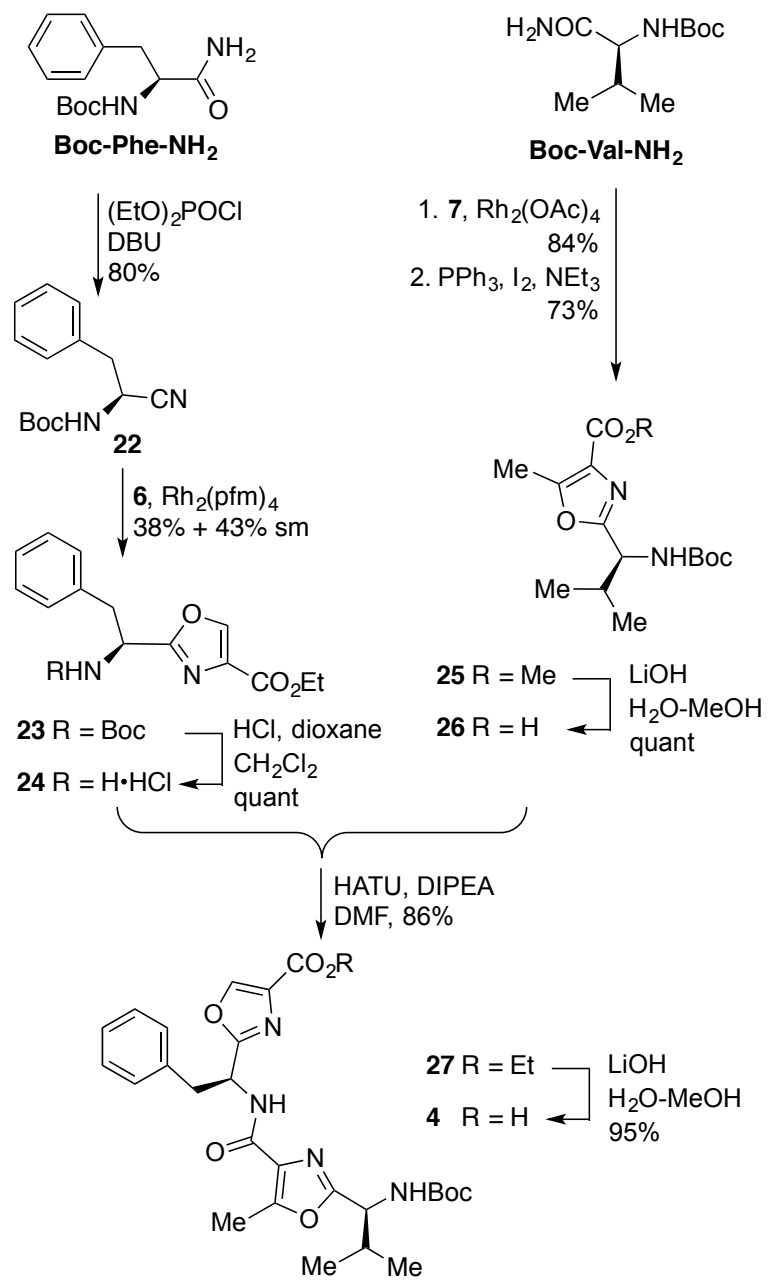

Towards the synthesis of wewakazole B, Boc-alaninamide and Boc-phenylalaninamide were each subjected to the N-H insertion-cyclodehydration sequence to give oxazoles $\mathbf{2 8}$ and $\mathbf{3 0}$ respectively. Bocdeprotection of $\mathbf{2 8}$ and ester deprotection of 30, followed by coupling of the resulting amine 29 and acid 31 using HATU gave the bis-oxazole 32. Final hydrolysis gave the wewakazole B fragment $\mathbf{5}$ (Scheme 6).

With all the required fragments in hand, all that remained was to couple them together to synthesize both natural products. The bis-oxazole 4 was coupled to the octapeptide 3 using DMTMM to give the 
cyclization precursor 33 in 69\% yield. The termini were deprotected under basic (97\%) and acidic (quant) conditions respectively, and cyclization of the 36-membered ring, again using DMTMM as the coupling agent, delivered wewakazole 1 in $68 \%$ yield after 4 days at room temperature, at $0.55 \mathrm{mM}$ concentration. The rotamerism observed in the acyclic intermediates was entirely absent in the macrocycle, and good NMR spectra could be obtained. A systematic $0.3 \mathrm{ppm}$ difference in the ${ }^{13} \mathrm{C}$ NMR spectrum not-withstanding, the NMR spectroscopic data obtained for this material matched closely those reported for the natural product allowing us to confirm the structure of wewakazole.

Scheme 6. Synthesis of bis-oxazole fragment of wewakazole B.

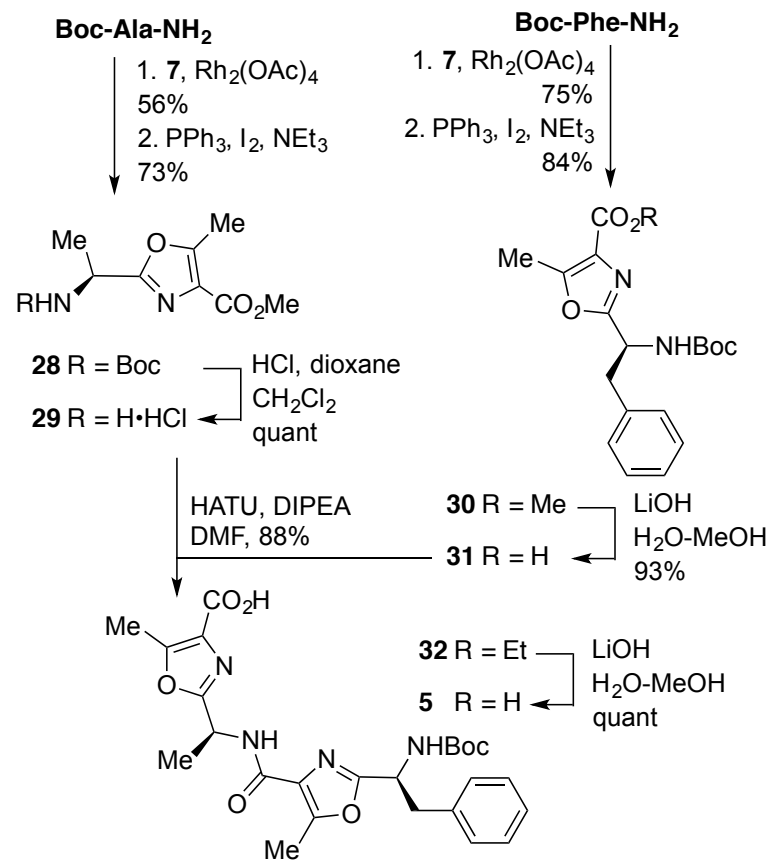

With wewakazole complete, attention turned to wewakazole B. Coupling of the octapeptide 3 with the bis-oxazole fragment 5 proceeded in 76\% yield using DMTMM in chloroform. The C-terminal was deprotected first (85\%), and then the Boc group was removed from the $N$-terminal using $\mathrm{HCl}$ in dioxane (quant). Cyclization was first attempted using the conditions reported by $\mathrm{Wu}$ et al., ${ }^{4}$ with HATU in dichloromethane. After 7 days, the product could be isolated in $36 \%$ yield, with only a trace of tetramethylurea proving difficult to remove. An attempt to use PyBOP as the coupling agent, to circumvent the presence of the urea side-product, gave excellent conversion but purification was again very difficult due to the co-elution of wewakazole B with the tripyrrolidinophosphine oxide side product. As above, the best results were obtained using DMTMM in chloroform, which gave a 78\% yield of clean material after five days at room temperature. The spectroscopic data obtained for synthetic wewakazole B matched the literature data for the natural product. 
Scheme 7. Completion of the synthesis of the wewakazoles.

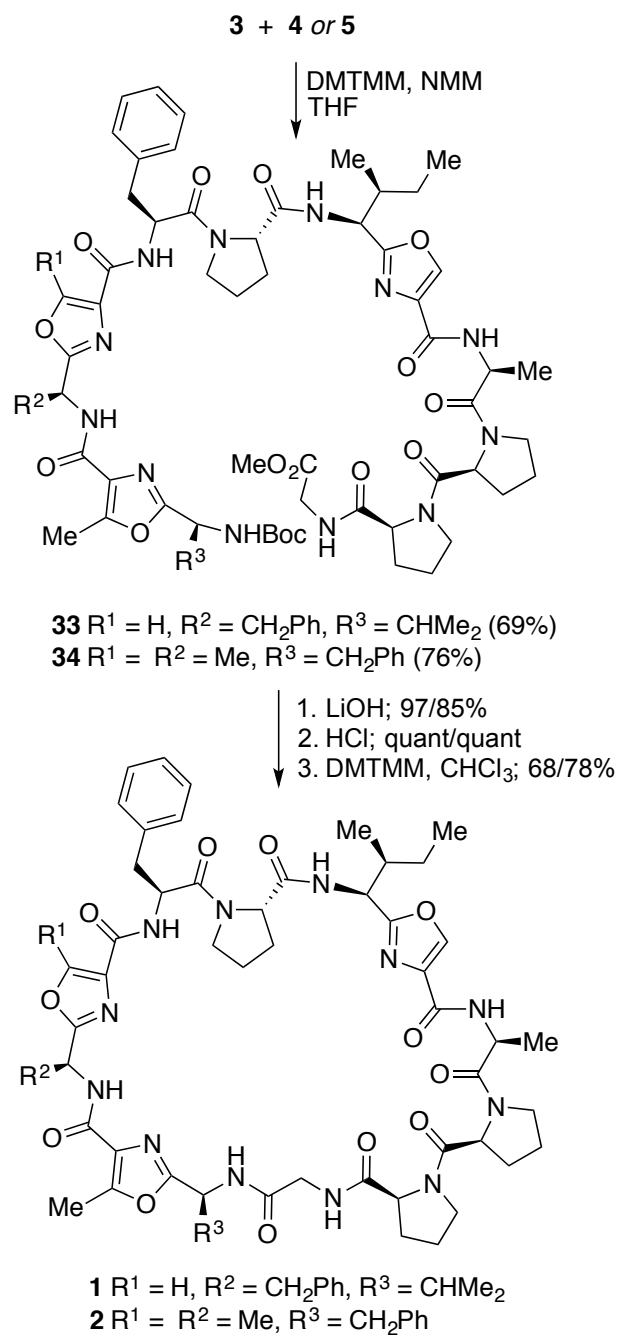

In summary, the total syntheses of wewakazole, and its congener wewakazole B have been carried out using rhodium catalyzed cycloadditions and $\mathrm{N}-\mathrm{H}$ insertion reactions to provide the oxazoles. The overall strategy is both convergent and divergent in nature, and can readily be applied to the synthesis of analogues of the natural products.

\section{ACKNOWLEDGMENT}

We thank the University of Nottingham for support.

\section{REFERENCES}

(1) Nogle, L. M.; Marquez, B. L.; Gerwick, W. H. Org. Lett. 2003, 5, 3-6.

(2) Malloy, K. L.; Villa, F. A.; Engene, N.; Matainaho, T.; Gerwick, L.; Gerwick, W. H. J. Nat. Prod. 2011, 74, 95-98.

(3) Lopez, J. A. V.; Al-Lihaibi, S. S.; Alarif, W. M.; Abdel-Lateff, A.; Nogata, Y.; Washio, K.; Morikawa, M.; Okino, T. J. Nat. Prod. 2016, 79, 1213-1218.

(4) Long, B. H.; Zhang, J. Z.; Tang, X. D.; Wu, Z. Z. Org. Biomol. Chem 2016, 14, 9712-9715.

(5) Nayani, K.; Hussaini, S. D. A. Tetrahedron Lett. 2017, 58, 1166-1169. 
(6) Bagley, M. C.; Bashford, K. E.; Hesketh, C. L.; Moody, C. J. J. Am. Chem. Soc. 2000, 122, 3301-3313.

(7) Hughes, R. A.; Thompson, S. P.; Alcaraz, L.; Moody, C. J. J. Am. Chem. Soc. 2005, 127, $15644-$ 15651.

(8) Linder, J.; Blake, A. J.; Moody, C. J. Org. Biomol. Chem. 2008, 6, 3908-3916.

(9) Wada, H.; Williams, H. E. L.; Moody, C. J. Angew. Chem. Int. Ed. 2015, 54, 15147-15151.

(10) Dexter, H. L.; Williams, H. E. L.; Lewis, W.; Moody, C. J. Angew. Chem. Int. Ed. 2017, 56, 3069-3073.

(11) Bagley, M. C.; Buck, R. T.; Hind, S. L.; Moody, C. J. J. Chem. Soc., Perkin Trans. 1 1998, 591600.

(12) Moody, C. J.; Bagley, M. C. J. Chem. Soc., Perkin Trans. 1 1998, 601-607.

(13) Doyle, K. J.; Moody, C. J. Tetrahedron 1994, 50, 3761-3772.

(14) Linder, J.; Garner, T. P.; Williams, H. E. L.; Searle, M. S.; Moody, C. J. J. Am. Chem. Soc. 2011, 133, 1044-1051.

(15) Kunishima, M.; Kawachi, C.; Morita, J.; Terao, K.; Iwasaki, F.; Tani, S. Tetrahedron 1999, 55, $13159-13170$

(16) Wipf, P.; Miller, C. P. J. Org. Chem. 1993, 58, 3604-3606. 